\title{
Magnetoresistance of the thin film ferromagnetic nanoconstrictions
}

\author{
CHENG Hao*, YANG Wei, LIU Hong \& WANG LingJiang \\ School of Electronic and Information Engineering, Chengdu University, Chengdu 610106, China
}

Received June 18, 2011; accepted August 12, 2011

\begin{abstract}
The magnetoresistance and $I-V$ characteristics at different temperatures of the thin film ferromagnetic nanoconstrictions of variable width (from 20 to $250 \mathrm{~nm}$ ) and $10 \mathrm{~nm}$ thicknesses, fabricated by electron beam lithography and vacuum thin film deposition are compared. The magnetoresistance and resistance of the thin film ferromagnetic nanoconstrictions are not related to the width of the nanoconstrictions. Instead the resistance of the local nano-region in the middle of the thin film ferromagnetic nanoconstriction has only a minor role compared to that of the two microscale thin film ferromagnetic electrodes, which contribute the majority of the measured resistance. The magnetoresistances of the thin film ferromagnetic nanoconstrictions and a $0.2 \mathrm{~cm} \times 0.8$ $\mathrm{cm}$ thin ferromagnetic film deposited under the same conditions were also compared; the thin film ferromagnetic nanoconstrictions have higher magnetoresistances than the thin ferromagnetic film, which implies that the measured magnetoresistance of the thin film ferromagnetic nanoconstrictions comes mainly from the local nano-region in their centers. In conclusion, the measured magnetoresistance of the whole sample is similar to the anisotropic magnetoresistance, because the resistance of the two microscale thin film ferromagnetic electrodes is much higher than that of the local nano-region in the middle of the samples. Comparing the experimental results for the thin film ferromagnetic nanoconstrictions and the thin ferromagnetic film reveals that the magnetoresistance of the local nano-region in the middle of the sample is much higher than that of the two microscale thin film ferromagnetic electrodes attached to it.
\end{abstract}

thin film vacuum deposition, ferromagnetic nanoconstrictions, anisotropic magnetoresistance, ballistic magnetoresistance

Citation: Cheng H, Yang W, Liu H, et al. Magnetoresistance of the thin film ferromagnetic nanoconstrictions. Chin Sci Bull, 2012, 57: 445-449, doi: $10.1007 / \mathrm{s} 11434-011-4881-5$

Moore's Law states that the number of transistors on a chip has roughly doubled every two years since the integrated circuit (IC) was invented in 1959 and successfully applied in the semiconductor industry [1]. Fundamental research into the electronic devices is mainly focused on two areas. One is manipulation of single atoms and molecules to integrate an ultra-large-scale integrated circuit on the nanoscale $[2,3]$, where individual atoms or molecules can be used as the basic information unit [4]. The other is the electron spin [5], since by the manipulation of a single electron and electron spin together, the spin properties can be used instead of or in addition to the electron charge properties to carry information. In both these hot research areas, it is still extremely difficult to fabricate a stable conductive point con-

*Corresponding author (email: hchengkeke@yahoo.com) tact to a single atom or molecule; in contrast, fabrication of a stable conductive point nanocontact and nanoconstriction is much easier [6]. Ferromagnetic point nanocontacts and nanoconstrictions are effective tools for studying the interaction between magnetic field and electron spin. To understand the relationship between electron spin and electron charge in limited small ferromagnetic areas, it is important for us to study the spin-dependent electron transport dominated by quantum mechanics in such mesoscopic systems and the conductive behavior of the individual atoms and molecules. Thus ferromagnetic point nanocontacts and nanoconstrictions have become a research focus in recent years [7-12].

A nanocontact is a conductive channel between the surfaces of two crystallites, formed by pressure or other means. Generally, a magnetoresistance of more than $200 \%$ at room 
temperature can be observed in ferromagnetic nanocontacts fabricated by the mechanical, electrochemical or coldpressing methods $[7,9,12]$. This phenomenon is referred to as "ballistic magnetoresistance" (BMR), where a nanoscale or even atomic scale magnetic domain wall is presumed to be located in the ferromagnetic nanocontacts. The transmission probabilities of the spin-up and spin-down electrons are very different when the electrons are transported through the thin domain wall, and this contributes to the very large BMR ratios [13]. BMR generates a heated debate among scientists [14-16], because of a lack of effective experimental means to characterize and prepare the nanoscale domain wall. Another issue is structural defects, since the structure of the ferromagnetic nanocontacts fabricated by conventional methods is changed during the magnetoresistance measurements and the observed large ratio BMR may be caused by these structural changes. However, the fact that BMR can be observed in ferromagnetic nanocontacts fabricated by electrodepositing indicates that the BMR comes from the local nano-region in the middle of the nanocontacts, and is not associated with the macroelectrodes [17]. In any case, on one hand, ferromagnetic nanocontacts fabricated by a method which can avoid structural changes during magnetoresistance measurements will play a key role in the study of BMR; on the other hand, the BMR head may eventually replace the widely used giant magnetoresistance (GMR) head, since the magnetoresistance ratios observed for BMR are far beyond that of GMR at room temperature, which means that the ferromagnetic nanocontacts will need to be fabricated by a technology compatible with the modern semiconductor industry.

In this paper, the magnetoresistance and $I-V$ characteristics at different temperatures of thin film ferromagnetic nanoconstrictions of variable width (from 20 to $250 \mathrm{~nm}$ ) and $10 \mathrm{~nm}$ thickness fabricated by electron beam lithography and vacuum thin film deposition are compared systematically to obtain the relationship between the magnetoresistance and the width of the thin film ferromagnetic nanoconstrictions. To distinguish the magnetoresistance of the local nano-region in the middle of the sample from that of the two microscale thin film ferromagnetic electrodes, a $10 \mathrm{~nm}$ thick, $0.2 \mathrm{~cm} \times 0.8 \mathrm{~cm}$ thin ferromagnetic film was also prepared using the same vacuum conditions. The magnetoresistance of the thin ferromagnetic film is compared to that of the thin film ferromagnetic nanoconstrictions.

\section{Experimental methods}

The thin film ferromagnetic Permalloy nanoconstrictions were fabricated by combining electron beam lithography and vacuum thin film deposition techniques on a $\mathrm{Si}$ wafer. Firstly the Si substrate was covered with a double layer of LOR/PMMA by spin-coating, 30\% LOR 3A by weight as a bottom layer was spun at $2000 \mathrm{r} / \mathrm{min}$ for $60 \mathrm{~s}$ and then the resist baked at $180^{\circ} \mathrm{C}$ in an oven for $20 \mathrm{~min}$; $2.5 \%$ PMMA 2041 by weight as top layer was spun at $2000 \mathrm{r} / \mathrm{min}$ for $60 \mathrm{~s}$ and then the resist baked again at $180^{\circ} \mathrm{C}$ in an oven for an hour; secondly the LOR/PMMA covered Si wafer was exposed to prepare the different widths of nanoconstriction patterns using an e-beam writer (VB6 from Leica-Cambridge LTD) at $100 \mathrm{keV}$ with a dose from 500 to $2000 \mu \mathrm{C} / \mathrm{cm}^{2}$. Ti $(2 \mathrm{~nm}) / \mathrm{NiFe}(10 \mathrm{~nm}) / \mathrm{Ti}(2 \mathrm{~nm})$ thin film nanoconstrictions were then prepared by vacuum thin film deposition using a Nordiko DC magnetron sputtering system followed by liftoff process. A $10 \mathrm{~nm}$ thick, $0.2 \mathrm{~cm} \times 0.8 \mathrm{~cm}$ thin NiFe film on a $\mathrm{Si}$ wafer was also prepared at the same time. To facilitate measurements of the prepared thin film ferromagnetic nanoconstrictions, a second level photoresist layer was spincoated onto the surface of the sample, and then optical lithography and electron beam thermal evaporation were performed to prepare $320 \mathrm{~nm}$ thick, $1 \mathrm{~mm} \times 1 \mathrm{~mm} \mathrm{Cr}(20 \mathrm{~nm}) / \mathrm{Cu}$ $(300 \mathrm{~nm})$ macroelectrodes on top of each microscale thin film ferromagnetic electrode. A scanning electron microscope (SEM) and an atomic force microscopy (AFM) were used to characterize the morphology of the thin film ferromagnetic nanoconstrictions. The magnetoresistance and $I-V$ characteristics of the thin film ferromagnetic nanoconstrictions and the thin ferromagnetic films were measured with a measurement system which comprised a Keithley 2400 Sourcemeter, a commercial power driven bipolar electromagnet with magnetic field up to 6000 Oe and a commercial sample stage with a controllable temperature range from 80 to $500 \mathrm{~K}$. The whole integrated measuring system is controlled by a Labview graphical user interface and can run automatically.

\section{Results and discussion}

\subsection{Magnetoresistance and resistance of the thin film ferromagnetic nanoconstrictions of variable width in the range from $20 \mathrm{~nm}$ to $250 \mathrm{~nm}$}

Figures 1 and 2 are the morphology of prepared thin film ferromagnetic nanoconstrictions characterized by SEM and by AFM. A $20 \mathrm{~nm}$ wide thin film ferromagnetic nanoconstriction can be clearly seen in Figure 2. The nanoconstrictions with other widths were also characterized using SEM and AFM (not shown here); the results show that the width of the prepared thin film ferromagnetic nanoconstrictions is in the range from 20 to $250 \mathrm{~nm}$. Figures 3 and 4 are the magnetoresistance curves of a $20 \mathrm{~nm}$ wide thin film ferromagnetic nanoconstriction measured using a two-probe system with a DC current of $0.01 \mathrm{~mA}$ passed along the nanoconstriction at room temperature and $80 \mathrm{~K}$. The magnetic field is applied in the plane and perpendicular to the current in both cases. Two $10 \mathrm{~cm}$ long, $40 \mu \mathrm{m}$ diameter $\mathrm{Cu}$ cables are used to connect the two electrodes of the thin film ferromagnetic nanoconstrictions to the electrodes of the Keithley 2400 sourcemeter; one end of each $\mathrm{Cu}$ cable is 


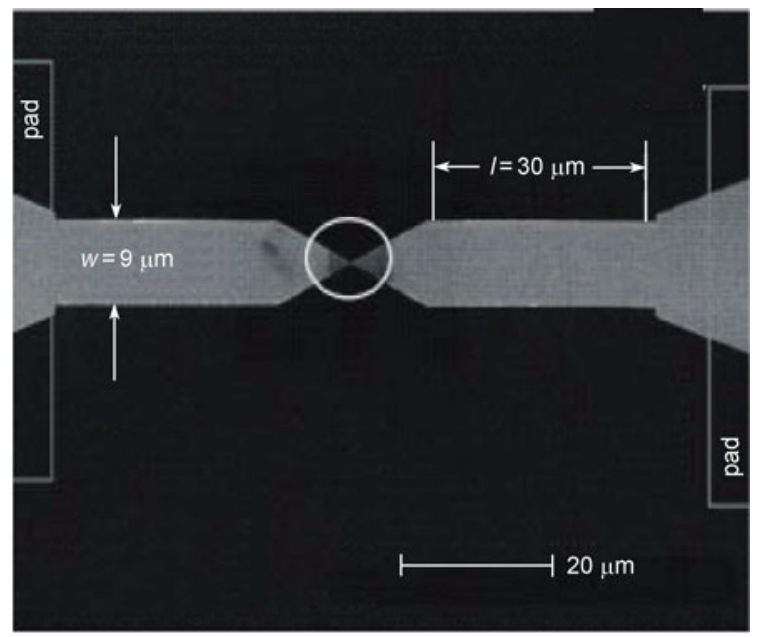

Figure 1 The morphology of a thin film ferromagnetic nanoconstriction characterized by SEM.

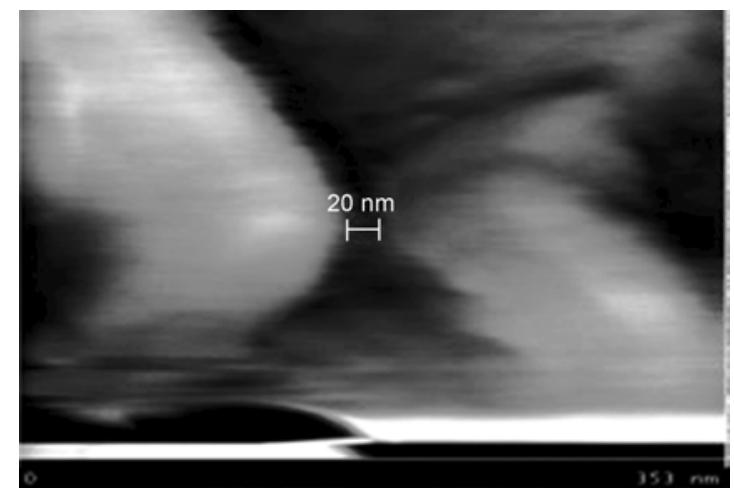

Figure 2 The morphology of the thin film ferromagnetic nanoconstriction characterized by AFM.

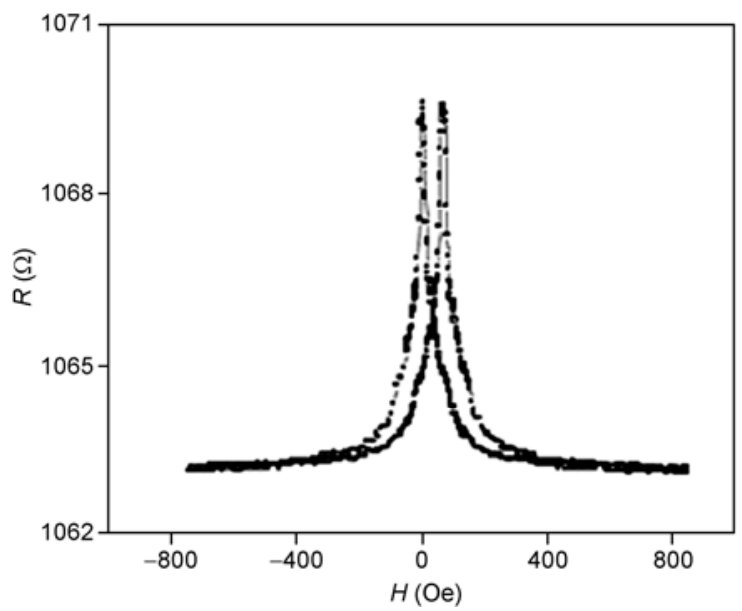

Figure 3 The magnetoresistance of a $20 \mathrm{~nm}$ wide, thin film ferromagnetic nanoconstriction measured at room temperature $(300 \mathrm{~K})$.

soldered to the electrodes of the sourcemeter, the other end of each cable is attached to one of the $1 \mathrm{~mm} \times 1 \mathrm{~mm} \mathrm{Cu}$ electrodes of the sample using conductive Ag adhesive. In

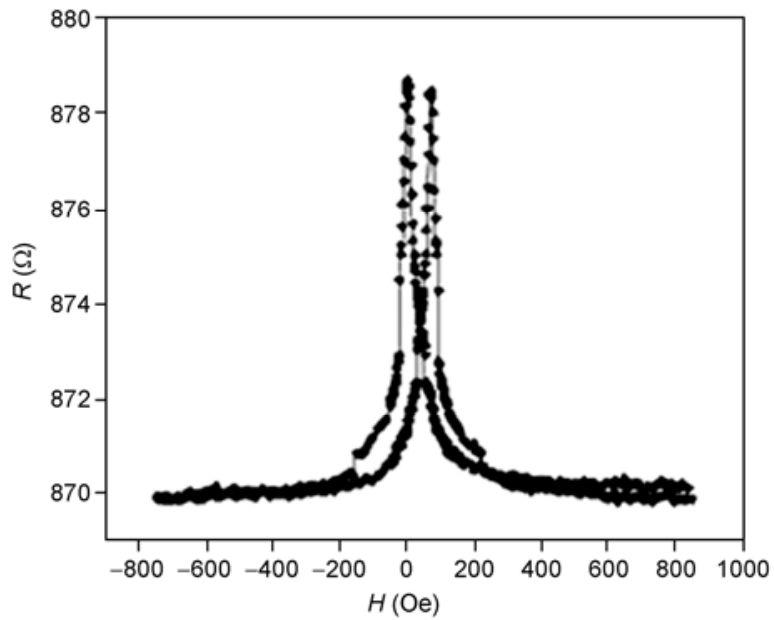

Figure 4 The magnetoresistance of a $20 \mathrm{~nm}$ wide, thin film ferromagnetic nanoconstriction measured at $80 \mathrm{~K}$

the two-probe arrangement, the measured resistance includes the resistance of the local nano-region in the middle of the sample, the two microscale thin film ferromagnetic electrodes, the two $\mathrm{Cu}$ cables and the two contacts between the $\mathrm{Cu}$ cables and the electrodes of the sample. Typically, the resistance of the $\mathrm{Cu}$ cables and the contacts between the cable and the electrode of the sample is less than $0.5 \Omega$, which is negligible compared to the resistance of the prepared thin film ferromagnetic nanoconstrictions at zero magnetic field, and thus the two-probe measurements are sufficiently precise for our purposes. The magnetoresistance of a $20 \mathrm{~nm}$ wide thin film nanoconstriction is only about $0.7 \%$ at room temperature and about $1 \%$ at $80 \mathrm{~K}$, as can be estimated from Figures 3 and 4. These two measurements show that anisotropic magnetoresistance (AMR) is the dominant contribution in the thin film ferromagnetic nanoconstrictions. To obtain the relationship between the magnetoresistance and the width of the thin film ferromagnetic nanoconstrictions, the magnetoresistance and resistance of the thin film ferromagnetic nanoconstrictions with widths from 20 to $250 \mathrm{~nm}$ at $80 \mathrm{~K}$ are shown in Figure 5. Both the magnetoresistance and resistance appear to be independent of the width of the thin film ferromagnetic nanoconstrictions. The $I-V$ characteristic for the $20 \mathrm{~nm}$ wide thin film ferromagnetic nanoconstriction measured at $80 \mathrm{~K}$ is shown in Figure 6 . The linear fit $I-V$ characteristic shows that the thin film ferromagnetic nanoconstriction is a normal metallic conductor. However given the dimension of the conductive channel in the nanoconstriction $(20 \mathrm{~nm} \times 10 \mathrm{~nm}$, as shown in Figures 1 and 2), quantum tunneling effects are expected, and these effects should cause the $I-V$ dependence to deviate from linear behavior towards a non-metallic conduction. This lack of quantum effects implies that the resistance of the local nano-region in the middle of the sample is much smaller than that of the two microscale thin film ferromagnetic electrodes attached to it, and the main 


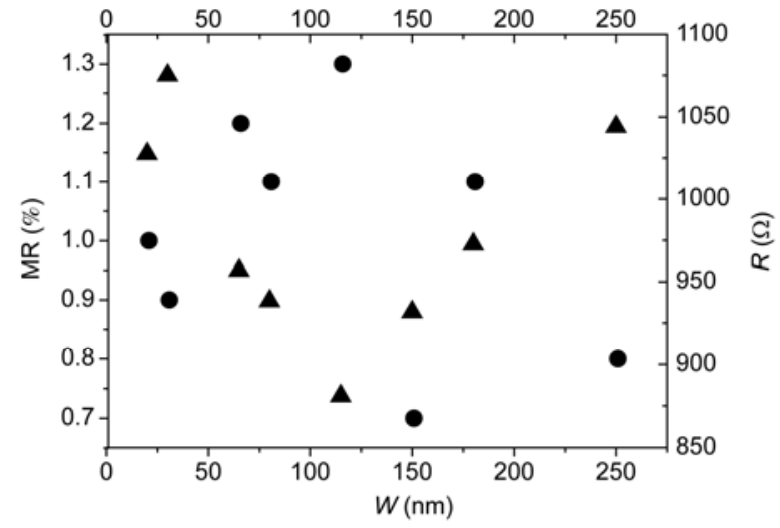

Figure 5 The measured resistance $(\boldsymbol{\Delta})$ and magnetoresistance $(\bullet)$ of thin film ferromagnetic nanoconstrictions of variable width (ranging from 20 to $250 \mathrm{~nm})$ at $80 \mathrm{~K}$

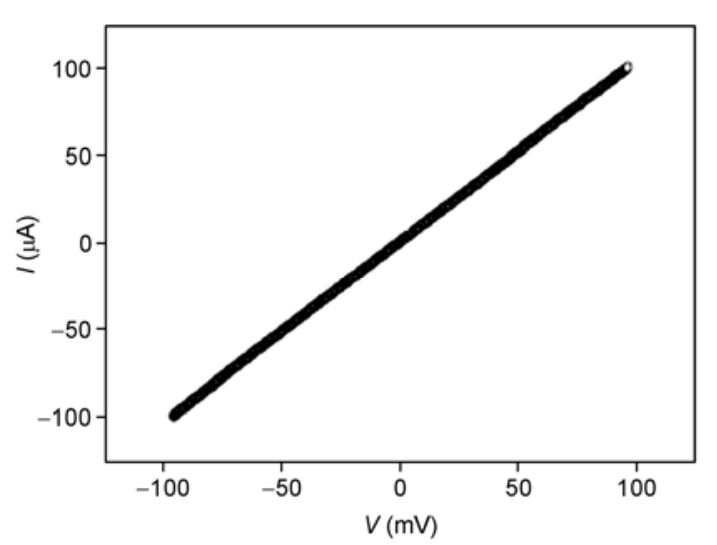

Figure 6 The $I-V$ characteristics of a $20 \mathrm{~nm}$ wide thin film ferromagnetic nanoconstriction measured at $80 \mathrm{~K}$.

contribution to the measured resistance must come from the two microscale thin film ferromagnetic electrodes. In short, we can conclude that the measured resistance is essentially the diffusive resistance of the two microscale thin film ferromagnetic electrodes. However we still cannot judge which component of the sample is the main contributor to the measured magnetoresistance.

\subsection{Comparison of the magnetoresistance of the thin film ferromagnetic nanoconstrictions and the thin fer- romagnetic films}

To clarify the contributions of the two components of the thin film ferromagnetic nanoconstrictions to the measured magnetoresistance, a four-probe measurement was used to measure the magnetoresistance of a $0.2 \mathrm{~cm} \times 0.8 \mathrm{~cm}$ thin ferromagnetic film deposited using the same conditions as for the thin film ferromagnetic nanoconstrictions. Figure 7 is the magnetoresistance of this thin ferromagnetic film measured with a DC current of $0.1 \mathrm{~mA}$ at room temperature. The magnetic field is applied in the plane and perpendicular

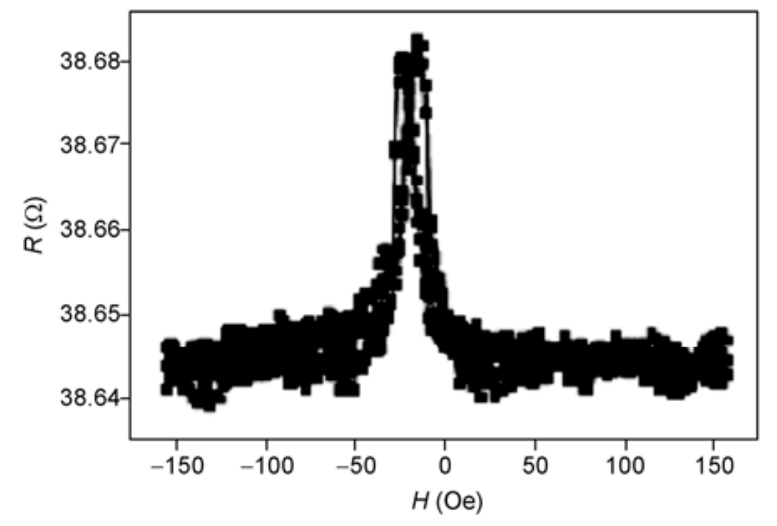

Figure 7 Magnetoresistance of a $0.2 \mathrm{~cm} \times 0.8 \mathrm{~cm} \times 10 \mathrm{~nm}$ thin ferromagnetic film at room temperature.

to the current. The magnetoresistance of the thin ferromagnetic film is about $0.1 \%$, which is much smaller than that of the thin film ferromagnetic nanoconstrictions. In our analysis, we assume that the resistivity and magnetoresistance of the two microscale thin film ferromagnetic electrodes are the same as those of the thin ferromagnetic film. The resistance of the $0.2 \mathrm{~cm} \times 0.8 \mathrm{~cm} \times 10 \mathrm{~nm}$ thin film is around $38.64 \Omega$ as shown in Figure 7; its calculated resistivity is $1.54 \times 10^{-4} \Omega \mathrm{cm}$. This can be combined with the dimensions of the two microscale thin film ferromagnetic electrodes, $2 \times 30 \mu \mathrm{m} \times 9 \mu \mathrm{m} \times 10 \mathrm{~nm}$ (referred to Figure 1), to calculate the resistance at zero magnetic field of the local nano-region in the middle of the thin film magnetic nanoconstrictions (29 $\Omega$ ), and its change (5.4 $\Omega$ ) upon application of the magnetic field. This means that the magnetoresistance of the local nano-region is $18.6 \%$. From the experiments, we can see that the measured resistance of the samples comes mainly from the two microscale thin film electrodes but the measured magnetoresistance comes mainly from the local nano-region in the middle of the samples. Since the resistance of the two microscale thin film ferromagnetic electrodes is much higher than that of the local nano-region in the middle of the samples, the overall measured magnetoresistance is very small and similar to the anisotropic magnetoresistance measured in the thin ferromagnetic films. Through the experiments on the analogous materials, we were able to show that the magnetoresistance of the local nano-region in the middle of the samples can reach $18.6 \%$.

\section{Conclusion}

Thin film ferromagnetic nanoconstrictions as small as 20 $\mathrm{nm}$ have been successfully prepared by electron beam lithography and vacuum thin film deposition. The resistance of the two microscale thin film ferromagnetic electrodes is much higher than that of the local nano-region in the middle of the samples; our experiments revealed that the measured 
magnetoresistance comes mainly from the local nano-region in the middle of the samples. Comparing measurements of the thin film ferromagnetic nanoconstrictions and the thin ferromagnetic film shows that the magnetoresistance of the local nano-region in the thin film ferromagnetic nanoconstrictions can reach $18.6 \%$, which is much higher than the anisotropic magnetoresistance of the thin ferromagnetic film.

1 Moore G E. Gramming more component onto integrated circuits. Electronics, 1965, 38: 114-117

2 Eigler D M, Schweizer E K. Positioning single atoms with a scanning tunneling microscope. Nature, 1990, 344: 524-526

3 Stroscio J A, Eigler D M. Atomic and molecular manipulation with the scanning tunnelling microscope. Science, 1991, 254: 1319-1326

4 Aviram A, Ratner M A. Molecular rectifiers. Chem Phys Lett, 1974, 29: 277-283

5 Wolf S A, Awschalom D D, Buhrman R A, et al. Spintronics: A spinbased electronics vision for the future. Science, 2001, 294: 14881495

6 Champagne A R, Pasupathy A N, Ralph D C. Mechanically adjustable and electrically gated single-molecule transistors. Nano Lett, 2005, 5: 305-308

7 Garcia N, Munoz M, Zhao Y W. Magnetoresistance in Ni nanocontacts in excess of $200 \%$ at room temperature and 100 Oe field. Phys
Rev Lett, 1999, 82: 2923-2926

8 Chopra H D, Hua S Z. Ballistic magnetoresistance over $3000 \%$ in $\mathrm{Ni}$ nanocontacts at room temperature. Phys Rev B, 2002, 66: 020403

9 Chopra H D, Matthew R S, Jason N A, et al. The quantum spin-valve in cobalt atomic point contacts. Nat Mater, 2005, 4: 832-837

10 Haug T, Perzlmaier K, Back C H. In situ magnetoresistance measurements of ferromagnetic nanocontacts in the Lorentz transmission electron microscope. Phys Rev B, 2009, 79: 024414

11 Sangiao S, Morellón L, Ibarra M R, et al. Ferromagnet-superconductor nanocontacts grown by focused electron/ion beam. Solid State Commun, 2011, 151: 37-41

12 Cheng H, Liu H, Wang L J, et al. Magnetoresistance of the ferromagnetic point nanocontacts (in Chinese). J Anhui Univ (Nat Sci Ed), 2011, 35: 64-69

13 Tatara G, Zhao Y W, Munoz M, et al. Domain wall scattering explains $300 \%$ ballistic magnetoconductance of nanocontacts. Phys Rev Lett, 1999, 83: 2030-2033

14 Egelhoff Jr W F, Gana L, Ettedguia H, et al. Artifacts that mimic ballistic magnetoresistance. J Magn Magn Mater, 2005, 287: 496-500.

15 Doudin B, Viret M. Ballistic magnetoresistance? J Phys: Cond Matter, 2008, 20: 083201

16 Mallett J J, Svedberg E B, Ettedgui H, et al. Absence of ballistic magnetoresistance in $\mathrm{Ni}$ contacts controlled by an electrochemical feedback system. Phys Rev B, 2004, 70: 172406

17 Garcia N, Munoz M, Qian G G, et al. Ballistic magnetoresistance in a magnetic nanometer sized contact: An effective gate for spintronics. Appl Phys Lett, 2001, 79: 4550-4552

Open Access This article is distributed under the terms of the Creative Commons Attribution License which permits any use, distribution, and reproduction in any medium, provided the original author(s) and source are credited. 